\title{
Questes
}

Revue pluridisciplinaire d'études médiévales

\section{Entre humanité et bestialité : Riccold de Montecroix face à l'autre dans son voyage}

\section{Marco Robecchi}

\section{(2) OpenEdition}

Journals

\section{Édition électronique}

URL : http://journals.openedition.org/questes/4393

DOI : 10.4000/questes.4393

ISSN : 2109-9472

\section{Éditeur}

Les Amis de Questes

\section{Édition imprimée}

Date de publication : 31 mars 2017

Pagination : $65-80$

ISSN : 2102-7188

\section{Référence électronique}

Marco Robecchi, «Entre humanité et bestialité : Riccold de Montecroix face à l'autre dans son voyage », Questes [En ligne], 35 | 2017, mis en ligne le 15 avril 2017, consulté le 30 avril 2019. URL : http:// journals.openedition.org/questes/4393 ; DOI : 10.4000/questes.4393 


\title{
Entre humanité et bestialité : Riccold de Montecroix face à l'autre dans son voyage
}

\author{
Marco ROBECCHI \\ Università degli Studi di Verona-Université Paris-Sorbonne
}

Les études médiévales postcoloniales nous ouvrent des champs de recherche dans lesquels nous devons encore nous engager, des territoires habités de populations médiévales peu familières, des langues et des cultures de genres très différents ${ }^{1}$.

À partir du XII ${ }^{\mathrm{e}}$ siècle, l'Occident (re)commence à voyager et, par conséquent, à rencontrer des peuples qu'il ne connaissait pas, ou qu'il ne connaissait qu'indirectement. Nous nous intéresserons dans cet article à la rencontre de Riccold de Montecroix avec les Turcs, les Tartares et les Kurdes, au cours de son voyage au Proche-Orient entre 1288 et $1300^{2}$.

Or, lorsque l'on s'intéresse au concept d'Orientalisme proposé par Edward Saïd, une question surgit immédiatement. Il affirme que, dès le Moyen Âge, les Occidentaux tendent à souligner leurs différences par rapport aux Orientaux : « l'Oriental devient plus Oriental, l'Occidental

\footnotetext{
${ }^{1}$ «Postcolonial medieval studies are thus opening up for us fields of inquiry into which we have yet fully to venture, territories populated by unfamiliar medieval peoples, languages, and cultures of many different stripes » (nous traduisons), Simon Gaunt, «Can the Middle Ages Be Post-Colonial ? », Comparative Literature 61/2, 2009, p. 170-176, cit. p. 175. Signalons que la bibliographie de cet article est très riche.

${ }^{2}$ Il s'agit d'un aspect qui n'a pas encore été suffisamment exploité. Je signale l'étude sur sa «théologie de l'Islam » par Rita George-Tvrtkovic, A Christian Pilgrim in Medieval Iraq: Riccoldo da Montecroce's Encounter with Islam, Turnhout, Brepols, coll. «Medieval voyaging», 2012, et l'étude sur son rapport avec les chrétiens orientaux par Camille Rouxpetel, «Riccoldo da Monte Croce's Mission towards the Nestorians and the Jacobites, (1288-c. 1300) », Medieval Encounters, 21, 2015, p. 250-268.
} 
plus Occidental $^{3} »$. Cette conception du rapport à l'autre limite la rencontre entre des cultures, des traditions et des sociétés différentes. Dans un texte du Moyen Âge où le narrateur en personne raconte son voyage en Orient et sa rencontre avec des Orientaux, deux questions au moins se posent. L'auteur effectuait-il une nette distinction entre lui, l'Occidental, et les autres, les Orientaux ? Et cette distinction empêchaitelle une ouverture de la pensée et du jugement dans son discours, quelle qu'elle soit ?

\section{Riccold de Montecroix}

Qui était Riccold de Montecroix (Riccoldo da Monte di Croce, en italien $^{4}$ ) ? Nous possédons peu d'informations à son sujet, et ce que l'on sait est tiré du Nécrologe de Santa Maria Novella, le couvent dominicain de Florence auquel il avait consacré sa vie. Mort en 1320, il était probablement entré dans l'ordre en $1267^{5}$. Sa formation intellectuelle «solide et même approfondie ${ }^{6}$ » se déroula dans le couvent de Santa Maria Novella : il avait donc une bonne connaissance de la théologie et des outils dominicains de prédication.

Après avoir débarqué à Acre en 1288, il passa quelques mois en Terre sainte avant de commencer sa tâche de missionnaire. Il avait été envoyé en mission par le pape Nicolas IV et par le maître général de l'Ordre Muño de Zamora, selon ce qu'il affirme dans son récit : «Donc,

\footnotetext{
${ }^{3}$ "The Oriental becomes more Oriental, the Westerner more Western " (nous traduisons), Edward Saïd, Orientalism [1978], London, Penguin, 2003, p. 45-46.

${ }^{4}$ Le nom Monte Croce est « un nom attaché à la famille Pennino [...] originaire d'un village de la campagne voisine, appelé Monte Croce », René Kappler, Riccold de Monte Croce. Pérégrination en Terre Sainte et au Proche-Orient. Lettres sur la chute de Saint-Jean d'Acre, Paris, Honoré Champion, coll. «Textes et traductions des classiques français du Moyen Âge », 1997, p. 13.

5 Pour la formation de Riccold et ses premières années, voir aussi Rita George-Tvrtkovic, A Christian Pilgrim..., op. cit., p. 2-7.

${ }^{6}$ René Kappler, Riccold..., op. cit., p. 13.
} 
lorsque j'eus reçu mon obédience de Monseigneur le pape, par le maître de l'Ordre, je me mis en route ${ }^{7} \gg$. Il sillonna d'abord la Terre Sainte avant de voyager en Anatolie et au Proche Orient jusqu'à la cour de Bagdad, où régnait le Khan des Mongols depuis la chute du Califat en 1258, pour y demeurer plusieurs années. Dans cette même ville, en 1291, il reçut la nouvelle de la chute de Saint-Jean d'Acre, la dernière forteresse chrétienne en Orient: cet épisode déclencha la rédaction des cinq Epistolae ad Ecclesiam triumphantem, où il s'interroge sur la Salvation et plaint le sort de la chrétienté. Encore à Bagdad il étudia l'arabe, ce qui lui permit d'avoir un accès direct au Coran ${ }^{8}$. Il fut l'un des rares Européens à résider longuement à la cour du Khan Mongol, ou du moins à avoir raconté son expérience. Il est fort probable qu'il ait écrit son récit, le Liber peregrinationis, peu après son retour à Florence, dans une période de création intense, puisqu'il produisit deux autres œuvres: le Contra legem sarracenorum, une œuvre polémique contre l'Islam, et le Ad nationes Orientales, une œuvre polémique contre les églises d'Orient.

\section{Les Sarrasins dans le Liber peregrinationis}

Son récit intitulé Liber peregrinationis ${ }^{9}$ raconte son voyage d'Acre jusqu'à Bagdad. Il faut remarquer que sa description est enrichie par plusieurs détails géographiques, anthropologiques, ethnographiques et religieux inédits et de grand intérêt. Le prologue, dans lequel l'auteur explique ses intentions, est à ce titre tout à fait significatif : «Il [mon récit] contient, brièvement, les royaumes, les peuples, les provinces, les

\footnotetext{
${ }^{7}$ René Kappler, Riccold..., op. cit., p. 38 « Suscepta igitur obedientia domini pape mediante magistro ordinis incipiens peregrinationem ».

${ }^{8}$ Une copie du Coran, avec les notes de lecture en latin de Riccold, est aujourd'hui conservée à la Bibliothèque nationale de France, sous la cote ar. 384.

${ }^{9}$ Selon l'incipit que l'on lit dans les manuscrits Incipit liber peregrinationis, voir René Kappler, Riccold..., op. cit., p. 36.
} 
lois, les rites, les sectes, les hérésies et les monstres que j'ai trouvés dans les régions de l'Orient $^{10}{ }$.

On peut diviser l'ouvrage en trois parties. La première décrit son pèlerinage en Terre sainte proprement dit, lequel pourrait être considéré comme la vraie peregrinatio ad loca sancta. La deuxième décrit le voyage depuis Acre jusqu'à Bagdad, en passant par Tyr, Sidon, Tripoli, Tarsus, Sivas, Erzurum, Tabriz, Ninive et Mossoul, où il rencontre plusieurs peuples, notamment les Turcs, les Tartares, les Kurdes et les chrétiens orientaux, à savoir les Jacobins, les Maronites et les Nestoriens. Cette section correspond au véritable «voyage », qu'on pourrait appeler peregrinatio inter infidelibus. La dernière partie décrit sa rencontre avec les musulmans de Bagdad, qui deviennent son centre d'intérêt principal. Cette troisième partie est à son tour divisée en deux sections : dans la première, il loue les sept auvres de perfection des musulmans, tandis que dans la seconde il condamne six défauts de leur théologie ${ }^{11}$.

La réputation de Riccold est surtout liée à son activité $\mathrm{d}^{\prime}$ ' islamologue » avant l'heure et à son œuvre la plus célèbre, le Contra legem sarracenorum. D'après Monneret de Villard, Riccold représente «l'aboutissement de presqu'un siècle de recherches sur l'Islam ${ }^{12}{ }$,

\footnotetext{
${ }^{10}$ René Kappler, Riccold..., op. cit., p. 36: «contenitur [...] sub brevitate regna, gentes, prouincie, leges, ritus, secte et hereses et monstra que inueni in partibus orientis ».

${ }^{11}$ Kappler aussi parle d'un triptyque, mais il se borne à identifier les trois sections avec la description des Tartares, des chrétiens d'Orient et de l'Islam. Voir René Kappler, Riccold..., op. cit., p. 16-17. Rita George-Tvrtkovic, A Christian Pilgrim..., op. cit., p. 32 résume bien la question : "He believed that his trip to Baghdad was an extension of his Holy Land pilgrimage, the first part of which served as the necessary spiritual foundation for his overall journey » (nous traduisons : il croyait que son voyage vers Bagdad était une prolongation de son pèlerinage dans la Terre sainte ; cette première partie était le début spirituel nécessaire pour l'ensemble de son voyage).

${ }^{12}$ Ugo Monneret de Villard, Lo studio dell'Islam in Europa nel XII e nel XIII secolo, Città del Vaticano, Biblioteca Apostolica Vaticana, coll. «Studi e testi - Biblioteca Apostolica Vaticana », 1944, p. 1: "Il punto d'arrivo di una serie di indagini
} 
depuis l'époque où Pierre le Vénérable, vers le milieu du XII siècle, commanda la traduction du Coran et écrivit une analyse de la religion musulmane. Toutefois, l'élément qui rend l'œuvre de Riccold vraiment digne d'une attention particulière est certainement l'experientia qu'il revendique lorsqu'il parle de la religion de Mahomet : bien qu'il n'ait pas été le seul Européen à vivre parmi les musulmans durant une longue période, les autres auteurs «citent rarement leur expérience personnelle de cette façon ${ }^{13} »$. Et c'est précisément cette expérience qui déstabilise son jugement, qui fait surgir toutes les ambivalences de son rapport avec les musulmans, qui modèle, enfin, sa «théologie de l'Islam» sous le signe de la merveille, du doute et de la dissonance ${ }^{14}$.

\section{Les autres peuples : les Turcs, les Tartares et les Kurdes}

L'éloge des musulmans de Bagdad n'est pas isolé dans le Liber peregrinationis. En effet, les rencontres de Riccold avec les autres peuples orientaux sont également dignes d'intérêt. Notre réflexion sera limitée à sa rencontre avec les Turcs, les Tartares et les Kurdes, selon l'ordre de leur apparition dans le récit. Il faut d'abord remarquer que la présentation d'un peuple est la conséquence du déplacement géographique du frère, qui fait office d'introduction. Ainsi le chapitre des Turcs s'ouvre comme suit: «L'Arménie traversée, nous entrâmes en Turquie, et nous y trouvâmes les Turcomans »; le chapitre des Tartares ainsi : « À notre entrée en Turquie nous découvrîmes le peuple [...] des Tartares »; et enfin le chapitre des Kurdes de même : «De là, allant vers

sull'Islam, che occupano quasi un secolo e mezzo » (nous traduisons : le point d'arrivée d'une série d'enquêtes sur l'Islam qui occupent près d'un siècle et demi).

13 «rarely cite personal experience as such» (nous traduisons), Rita GeorgeTvrtkovic, A Christian Pilgrim..., op. cit., p. 15.

${ }^{14}$ Ibid., p. 108 «wonder, doubt and dissonance. » 
le sud, nous parvînmes [au peuple des] Curtes $^{15} »$. À l'exception des Tartares, qui sont nomades (ce qui sera expliqué par la suite), l'auteur identifie le peuple au territoire où celui-ci réside, conformément à la conception médiévale du monde : «Le critère géographique fonctionne comme une donnée objective de caractérisation de l'étranger. Il constitue le premier mode d'appréhension de l'autre procédant d'une assimilation entre la région et l'homme ${ }^{16} »$.

\section{Les Turcs}

Après avoir identifié ces peuples, il ajoute une brève description, construite avec des comparaisons récurrentes, puisées dans le monde animal. Ainsi les Turcs sont définis comme :

des hommes presque sauvages qui sont sarrasins et habitent d'ordinaire sous terre, comme les taupes. Ils sortent des cavernes creusées dans la terre, comme des rats [...]. Ils sont féroces et courageux, surtout les femmes ${ }^{17}$.

Peut-être l'auteur fait-il référence dans cet extrait aux troglodytes de la Cappadoce, qui vivaient en effet dans des cavernes creusées et aménagées pour devenir des habitations ${ }^{18}$. Les adjectifs bestiales, fortes et

15 René Kappler, Riccold..., op. cit., p. 76 «Armeniam autem transeuntes intrauimus in Turchiam et invenimus Turchymanos», p. 78 "Intrantes autem infra Turchiam invenimus [...] gentem Tartarorum», p. 118 «Inde progredientes ad meridiem venimus ad [...] gentem Curtorum ».

${ }^{16}$ Wilfrid Besnardeau, Représentations littéraires de l'étranger au XII siècle. Des chansons de geste aux premières mises en roman, Paris, Honoré Champion, 2007, p. 35 .

${ }^{17}$ René Kappler, Riccold..., op. cit., p. 76 «homines quasi bestiales qui sunt Sarraceni, et habitant communiter sub terra ad modum talparum. Isti egrediuntur de cauernis terre quasi mures [...]. Isti sunt homines feroces et fortes et maxime mulieres eorum ». La désignation Sarraceni contribue, bien que peut-être involontairement, à aggraver le ton péjoratif de cette description.

${ }^{18}$ À la fin du XIII siècle, la turquisation de l'Anatolie était déjà assez avancée. Peut-être Riccold considérait-il comme Turcomans les peuples encore indigènes, mais sujets aux Turcomans depuis plus de deux siècles. Voir Claude Cahen, La Turquie pré-ottomane, Istanbul, Divit Matbaacilik Ve Yayincilik, coll. «Varia Turcica », 1991, en part. p. 104-110. 
feroces insistent sur l'image négative qu'il donne de ce peuple. Néanmoins, après ce jugement négatif prononcé à propos des Turcs, Riccold ajoute deux épisodes qui montrent son émerveillement et, nous semble-t-il, le doute qui l'étreint, ou du moins une sorte de dissonance, par rapport à ce jugement négatif, ce qui jette une lumière nouvelle sur sa description. Après avoir introduit le passage concernant les femmes «surtout les femmes, et je ne rapporterai qu'un trait à ce sujet [...]», il décrit une femme enceinte qui, suivant à pied les chameaux dans le désert, accoucha en silence dans la nuit: «Or au matin on vit avec étonnement que la mère avait pris l'enfant sur elle et suivait les chameaux, comme avant ${ }^{19} \gg$. L'adjectif admirantes (avec étonnement), témoigne d'un point de vue différent sur le concept de bestialité qu'il vient d'évoquer: ce n'est plus une bestialité effrayante, mais une bestialité qui suscite l'admiration pour sa puissance surhumaine, et ce d'autant plus qu'elle se manifeste chez une femme. Le fait qu'il ait décidé d'en raconter seulement un exemple semble sous-entendre que Riccold en aurait plusieurs en mémoire.

L'autre épisode qu'il raconte a une portée plus large : il s'agit du rapport des Turcs et des Grecs, c'est-à-dire les Byzantins. Les Grecs, orthodoxes, avaient fait du tort aux Francs, latins, « durant la désastreuse croisade de Conrad III $(1147)^{20} »$, en mêlant de la chaux à la farine de leurs pains. Or, les Turcs haïssaient les Grecs au point de les tuer s'ils sortaient de leurs villes sans un licol (vraisemblablement un lien autour du cou, comme les bêtes) ; ceux qui portaient ce licol, étaient capturés et vendus comme esclaves. Ce qui retient l'attention ici est que Riccold considère l'attitude des Turcs comme le résultat d'une volonté divine,

\footnotetext{
${ }^{19}$ René Kappler, Riccold..., op. cit., p. 76 «maxime mulieres eorum et ut de ceteris sileam hoc unum referam [...] mane autem facto, admirantes invenerunt infantulum vagientem quem accipiens mater pedes sequebatur camelos ut prius ».

${ }^{20}$ René Kappler, Riccold..., op. cit., p. 79.
} 
«Et c'est ainsi qu'en vérité s'accomplit à leur sujet ce dit de Salomon : "le méchant est pris à ses propres méfaits, dans les liens de son péché il est capturé $^{21}$ " ». Les Turcs, musulmans et bestiaux, deviennent donc pour le frère dominicain l'instrument de la justice divine : il n'attribue plus cette vengeance à leur bestialité. Au-delà de la question divine, Riccold se met-il du côté des Turcs en condamnant les Grecs, qui, bien qu'étant chrétiens, ont trahi les Francs ? Le recours à la religion pourrait être un moyen de justifier sa prise de position, mais plus vraisemblablement il s'agit s'un sentiment et d'un jugement personnels.

\section{Les Tartares}

La question des Tartares, qui occupe presque un quart du récit, est inévitablement la plus complexe, et on se contentera ici d'en traiter seulement certains aspects. Ainsi le frère franciscain Guillaume de Rubrouck décrit sa rencontre avec les Tartares : «nous rencontrâmes les Tartares, et il me semblait d'entrer dans un autre monde ${ }^{22} »$.

Riccold les présente, tout d'abord, comme «le peuple horrible et monstrueux des Tartares ». Ensuite, il affirme qu' « ils diffèrent beaucoup de toutes les autres nations du monde par l'aspect, les mœurs et les rites $^{23} \gg$. Deux aspects sont particulièrement intéressants : les adjectifs orribilem et monstruosam dessinent les contours d'une inhumanité plus

${ }^{21}$ René Kappler, Riccold..., op. cit., p. 78 «sic uere completur in eis illud Salomonis: 'iniquitates sue capiunt impium, et funibus peccatorum suorum unusquisque constringitur' ». La citation biblique est tirée du Livre des proverbes 5, 22 : http://www.vatican.va/archive/bible/nova_vulgata/documents/novavulgata_vt_pr overbiorum_lt.html\#5, page consultée le 04/12/2015.

${ }^{22}$ «inuenimus Tartaros; inter quos cum intraui, uisum fuit mihi recte quod ingrederer quoddam aliud seculum» (nous traduisons). Guglielmo di Rubruk, Viaggio in Mongolia (Itinerarium), a c. di Paolo Chiesa, Milano, Fondazione Lorenzo Valla/Arnoldo Mondadori Editore, coll. «Scrittori greci e latini », 2011, p. 18.

${ }^{23}$ René Kappler, Riccold..., op. cit., p. 78 «orribilem et monstruosam gentem Tartarorum »; «differunt autem Tartari multum ab omnibus aliis nationibus de mundo ». 
nettement soulignée par le fait qu'ils sont différents de toutes les autres nations du monde et que, haïssant toute espèce de ville et d'habitation, ils se posent au-dehors de la civilisation humaine («ils semblent haïr toute espèce de cité et de maison d'habitation $\left.{ }^{24} »\right)$. En même temps, Riccold commence à leur attribuer des caractères animaux, comme il l'avait déjà fait avec les Turcs : les Tartares ont à la fois des visages « très semblables à des singes, surtout les vieillards ${ }^{25} »$, et, en ce qui concerne leur nomadisme, il l'interprète comme «une espèce d'instinct naturel, ils vivent comme les brutes et les oiseaux avec des migrations d'été et d'hiver ${ }^{26} »$. Il est frappant de constater que le nomadisme, hors du paradigme interprétatif de la civitas occidentale, devient une condition morale.

Toutefois, le fait qu'ils puissent croire en un Dieu, et non en plusieurs, semble troubler le discours de Riccold : «ils croient en effet à un Dieu et à une sorte de loi de nature ». Ensuite, peu après avoir présenté toutes ces caractéristiques négatives, il commence à remarquer des aspects positifs, par exemple :

ils haïssent fort le mensonge et aiment la vérité [...]. Lorsqu'ils écrivent aux grands de leur nation, ils éprouvent et observent un tel respect [...]. Ils jugent l'homicide très grave, et se gardent bien de tuer sans le motif le plus sérieux ${ }^{27}$.

\footnotetext{
${ }^{24}$ René Kappler, Riccold..., op. cit., p. 78 «videntur odire omnem civitatem et omne edificium habitationis ».

${ }^{25}$ René Kappler, Riccold..., op. cit., p. 78 «valde simile scimie, et maxime senes ».

${ }^{26}$ René Kappler, Riccold..., op. cit., p. 80 «instinctu autem quodam naturali uiuunt ut bestie et aues mutantes locum in estate et yeme ». Cf. Ilaria Sabbatini, " "Secondo saraino buono huomo et fedele" : la definizione del nemico tra topos e innovazione », Itineraria, 10, 2011, p. 75-99, où elle dit, page 90, " chi viveva al di fuori della società urbana si collocava più vicino alla natura animale che non a quella umana » (nous traduisons: ceux qui vivaient au dehors de la société urbaine étaient plus proches de la nature animale que de l'humaine).

${ }^{27}$ René Kappler, Riccold..., op. cit., p. 80 «credunt autem Deum et quandam legem nature » et p. 82-84 «mendacium multum odiunt et veritatem amant. Summe
} 
Bien qu'il ait commencé à peindre un tableau de monstruosité et de bestialité, Riccold ne manque pas de relever les aspects positifs qui semblent frapper et déstabiliser sa vision. Sa louange porte sur des qualités morales propres à la société civile, comme la veritas, l'honor, le respect pour les supérieurs - dans une société structurée hiérarchiquement - et le respect pour la vie, à savoir la condamnation de l'homicide sine maxima causa. Voilà que dans le tableau obscur de violence et d'inhumanité qu'il avait dessiné, des éléments d'humanité et de civilisation inattendus commencent à poindre. Les Tartares monstruosi apparaissent à présent comme une civilisation, ou pour être plus précis, comme une société attachée aux valeurs fondamentales. En décrivant leur croyance en une «vaine résurrection», tout à fait différente de la résurrection chrétienne, il affirme «Car la position des Tartares sur la résurrection est très proche de l'erreur où sont les Sarrasins et les juifs dans leur attente de la résurrection ${ }^{28} »$. Mutatis mutandis, il nous semble que le fait de rapprocher leur croyance, perçue comme exotique, à des grilles de lecture préalables soit une marque où il est possible de se reconnaître ${ }^{29}$.

diligunt honorem [...] Cum scribunt suis maioribus tantam reuerentiam habent et seruant [...] homicidium multum reputant et ualde cauent ut non occidant sine maxima causa ».

${ }^{28}$ René Kappler, Riccold..., op. cit., p. 90 «resurrectionem fatuam [...] positio autem Tartarorum de resurrectione ualde similis est et propinqua errori Sarracenorum et Iudeorum de resurrectione quam expectant ».

${ }^{29}$ Dans un certain sens, il semble considérer Chrétiens, Musulmans et Juifs comme une unité à laquelle se rapporter, puisqu'il lui manque d'autres termes de comparaison. "The farther from home, a more collective 'we' can be used of Christian and Muslims », Simon Gaunt, Marco Polo's Le Devisement du Monde, Narrative Voice, Language and Diversity, Turnhout, Brepols, 2013, p. 139 (nous traduisons : plus on s'éloigne, plus on peut employer un «nous» collectif pour désigner Chrétiens et Musulmans). 
Plus loin dans le récit, il ne manque pas de présenter les baxites ${ }^{30}$ comme «certains prêtres des idoles : ce sont des Indiens très savant aux mœurs très réglées et rigoureuses ${ }^{31} »$. Bien qu'ensuite il tienne à remarquer leurs erreurs par rapport à la foi chrétienne et à blâmer leur connaissance des arts de la magie, il est admirable qu'il souligne leur sapientia et leur moralité. L'emploi du terme pontifices pour désigner leur rôle est tiré du monde religieux occidental. Sans élargir l'analyse à d'autres passages du récit, cet indice est suffisant pour affirmer que, chez Riccold comme chez Marco Polo, «toutes les pratiques religieuses décrites [...] suggèrent que les praticiens de ces religions exotiques sont à bien des égards semblables à nous ${ }^{32} »$. Il s'agit donc de reconnaître son identité dans l'étranger oriental pour le décrire de façon compréhensible et pour y retrouver quelque chose de familier, de bien connu ${ }^{33}$.

En outre, il se situe du côté des Tartares quand ils se trouvent face au Calife, en affirmant qu' "Ils se saisirent de lui et lui firent subir une condamnation extrêmement rigoureuse, mais juste ${ }^{34} »$. Cette affirmation fait référence à la conquête de Bagdad par les Mongoles. Une fois le Calife emprisonné, ils lui demandent «Puisque tu ne manges pas d'or, mais du pain comme les autres hommes, pourquoi avoir amassé tant d'or

${ }^{30}$ Le terme bakhshi «à l'époque peut déjà designer des médecins bouddhistes, mais encore des chamans et d'autres personnages ». Jean-Paul Roux, Histoire de l'Empire mongol, Paris, Fayard, 1993, p. 414.

${ }^{31}$ René Kappler, Riccold..., op. cit., p. 92 «quosdam pontifices ydolorum et sunt indianis homines ualde sapientes et ualde ordinati et graues in moribus ».

${ }^{32}$ Simon Gaunt, Marco Polo's..., op. cit., p. 137 « everything about the religious pratices described [...] is conveyed by suggesting that these practitioners of exotic other religions are in many respects just like ourselves » (nous traduisons).

${ }^{33}$ Même s'il ne s'agit pas d'une manière de s'approprier l'Autre, on retrouve une conclusion similaire dans Bernal Díaz au $\mathrm{XVI}^{\mathrm{e}}$ siècle pendant la conquête de l'Amérique du Sud : voir Stephen Greenblatt, Marvelous Possessions. The Wonder of the New World, Chicago/Oxford, University of Chicago press, 1991, en part. p. 128-145.

${ }^{34}$ René Kappler, Riccold..., op. cit., p. 108 «comprehendentes autem eum fecereunt cum eo durissimum sed iustissimum iudicium ». 
qui suffirait au monde entier ${ }^{35}$ ? » et ensuite ils l'obligent à boire de l'or fondu pour condamner son avarice et son accumulation de richesses.

\section{Les Kurdes}

La dernière rencontre que l'on analysera est celle avec les Kurdes, appelés par Riccold Curti. Présenté comme « un peuple monstrueux et enragé, les Curtes, dépassent en malice et en sauvagerie toutes les autres nations barbares que nous trouvâmes ${ }^{36}$ ». Une fois encore, les adjectifs monstruosam et rabiosam, ainsi que les substantifs malitia et feritas, renvoient au monde animal ${ }^{37}$. La comparaison animale se poursuit : «Ils habitent dans des montagnes et des lieux abrupts, comme des chèvres sauvages ». Même le nom de ce peuple indiquerait sa malice et sa bestialité : «Ils sont appelés 'Curti' non parce qu'ils seraient de petite taille [...] mais 'Curti' en langue persane signifie 'loups'». Selon Kappler : «c'est en turc et non en persan que qûrd ou qûrt signifie loup. Étymologie d'ailleurs sans fondement ${ }^{38} »$. Sans entrer dans la question de la véritable origine du nom, il est évident que, si Riccold juge utile de

\footnotetext{
${ }^{35}$ René Kappler, Riccold..., op. cit., p. 110 «Ex quo non comedis aurum sed panem sicut ceteri homines, quare tantum aurum congregasti quod toti mundo sufficeret?».

${ }^{36}$ René Kappler, Riccold..., op. cit., p. 118 «monstruosam et rabiosam gente Curtorum qui excedunt in malitia et feritate omnes barbaras nationes qua inuenimus ».

${ }^{37}$ D'ailleurs, le mot monstra était déjà employé dans le prologue. On ne manquera pas de remarquer à ce propos qu'il est étonnant qu'il n'emploie pas le mot mirabilia dans la description des Tartres (voir ci-dessus) et dans le chapitre consacré à la Perse, où il affirme «inter alia monstra inuenimus siluestrem asinum Indie qui ab aliquibus reputatur honager ». René Kappler, Riccold..., op. cit., p. 118 «entre autres prodiges, nous vîmes un âne sauvage que certains considèrent comme un onagre ». Le mot monstrum et ses dérivations sont donc utilisés par Riccold pour indiquer quelque chose de bestial, d'inhumain, et confirment la vision animale qu'il a de ces peuples.

${ }^{38}$ René Kappler, Riccold..., op. cit., p. 119, n. 185 ; les citations se trouvent dans la même page 119 : Habitant autem in montibus et in preruptis locis sicut capree siluestres [...]. Dicuntur autem Curti non quia sint brevis stature [...] sed Curti in lingua persica sonat lupi ».
} 
rapporter cette étymologie, c'est parce qu'elle était utile à sa description animalisée ${ }^{39}$.

Toutefois, de la même manière que dans les deux autres exemples, sa description et sa vision sont troublées par un épisode qui lui fait prononcer le contraire de ce qu'il vient d'affirmer : «Dieu convertit leur rage en mansuétude à notre égard : ils se sont montrés très humains avec nous $^{40} »$. Par la suite, il décrit comment les Kurdes sauvèrent ses amis perdus dans le désert, comment ils les retirèrent des neiges, comment ils allumèrent des feux pour les réchauffer et comment ils leurs offrirent « du miel sauvage et de la manne du ciel $^{41} »$. Riccold ne s'attarde pas sur cet éloge et poursuit au contraire au sujet de «Trois péchés [qui] prospèrent parmi eux : l'homicide, le vol et la trahison. [Ils] ont encore beaucoup de traits de bestialité qu'il serait trop long de rapporter ${ }^{42} »$. Cependant il est évident que cet épisode, vécu par l'auteur en personne et donc fruit de son expérience, remet en question sa vision de l'autre, même du peuple considéré comme le plus bestial.

${ }^{39}$ En ce qui concerne leur religion il affirme « isti sunt Sarraceni et recipiunt Alcoranum, et multum odiunt Christianos [...] in quorum occisione maxime crassantur », René Kappler, Riccold..., op. cit., p. 120 : « Ils sont Sarrasins, suivent le Coran, et haïssent fort les Chrétiens [...] surtout les religieux qu'ils s'acharnent à tuer».

${ }^{40}$ René Kappler, Riccold..., op. cit., p. 120 «horum rabiem conuertit Deus nobis in mansuetudinem et multa humanitatem perstiterunt nobis ».

${ }^{41}$ René Kappler, Riccold..., op. cit., p. 120 «mel siluestre et mana celi». Même Guillaume de Rubrouck, qui avait défini les Mongols comme des barbaros pleins de caractéristiques négatives et presque animales, avait cependant souligné leur hospitalité, comme l'a remarqué Stefano Pittaluga, «Lo sguardo dell'altro », Itineraria, 20, 2011, p. 23-32, en particulier p. 26.

${ }^{42}$ René Kappler, Riccold..., op. cit., p. 120 «in eis maxime uigent tria peccata, scilicet homicidium latrocidium et proditio [...] [et] multas alias bestialitates quas longum esset enarrare ». 


\section{Louer l'autre?}

Après cette analyse, il apparaît que la vision qu'a Riccold de l'autre est bien plus complexe qu'une distinction rigide entre nous et l'étranger. La rencontre de Riccold n'a pas la profondeur que Simon Gaunt relève chez Marco Polo, il ne va pas jusqu'à retrouver the uncanny, l'inquiétante étrangeté que le Vénitien avait sentie aux bornes du monde. Le fait que les Kurdes aient été capables de manifester de l'humanité indiquerait qu'il ait retrouvé dans cet autre bestial quelque chose de luimême, de sa civilisation. Si l'inquiétante étrangeté provoque l'angoisse, Riccold recule devant cette angoisse et la canalise vers la volonté divine, en annulant son effet ${ }^{43}$. Certainement, sa formation dominicaine a influencé sa façon d'approcher le monde qu'il décrit. D'une part, il en tire un regard qu'on pourrait qualifier de « scientifique », parce qu'il structure son discours et ne voit pas de merveilles surnaturelles dans l'Orient ${ }^{44}$. Cependant, sa formation et sa vocation missionnaire finissent sans doute par renforcer sa vision du monde oriental, différent du monde occidental et objet de sa mission de conversion. Il pose des barrières très nettes entre lui et les autres : lui, Riccold, appartient à la civilisation, à la Chrétienté, tandis que les autres n'appartiennent pas au monde chrétien, à la civilisation, et donc sont relégués dans le domaine du monde animal, bestial et monstrueux.

Il faut d'abord remarquer la normalisation du discours qui pose des barrières entre le nous occidental et l'autre oriental, l'étranger, constituée par des formules que Riccold utilise ailleurs, plus ou moins variées mais tout de même récurrentes : horribilem, monstruosam, bestialem.

\footnotetext{
${ }^{43}$ Voir à ce propos Simon Gaunt, «L'inquiétante étrangeté de la littérature de voyage en français au Moyen Âge », Medioevo Romanzo, 34/1, 2010, p. 57-81.

44 Les seules merveilles qu'il décrit, comme Marco Polo, sont «assez quotidienne[s], dans la mesure où elle[s] n[e sont] pas invraisemblable[s]. » Ibid., p. 73.
} 
Toutefois, étant donné que le voyageur aspire à fournir une description véridique, son jugement et ses affirmations formulés en introduction vacillent, et cela l'oblige à relever des irrégularités. Son regard se fait plus objectif parce qu'il est fondé sur son expérience: tandis que le préjugé l'oblige à constituer son monde de façon bipolaire, son expérience l'oblige à abattre les murs de ses préjugés : il parvient à en découvrir «Humanité, sentiment et réactions émotives ${ }^{45}$ ».

Quel est l'effet de ce dépassement des idées reçues ? Change-t-il la vision du monde rigidement divisée en deux parties que Riccold voulait représenter? Il semble que non. Après avoir proposé l'autre point de vue, il retourne à des schémas d'interprétation plus traditionnels en employant la justification de l'intervention divine: les Turcs accomplissent le dit biblique de Salomon, les Kurdes sont mus par la volonté de Dieu. Bien qu'il soit convaincu d'avoir rencontré des peuples bestiaux, il veille à ce qu'ils soient bien insérés dans le plan divin de l'histoire. Lorsqu'il souligne les qualités de ces peuples en recourant à la justification divine, ce n'est pas seulement la conséquence de l'emploi de pures formules rhétoriques, mais c'est la véritable pensée d'un frère dominicain avec une solide formation théologique.

Il est néanmoins remarquable qu'il présente malgré tout des éléments susceptibles de remettre en question sa vision du monde. Le résultat n'est pas une polarisation complète, au sens où l'entendait Saïd, mais une distinction plus nuancée. Les Orientaux n'en deviennent pas «plus orientaux », mais, comme ils montrent des éléments de civilisation et d'humanité, ils apparaissent étonnamment plus proches. Ainsi, plutôt que de parler d'une opposition effective entre nous et les autres, il

\footnotetext{
45 «Umanità, sentimento e reazioni emotive » (nous traduisons), Stefano Pittaluga, «Lo sguardo dell'altro », op. cit., p. 25. Ilaria Sabbatini, " "Secondo saraino..." », op. cit., p. 90, souligne comment le couple «selvatique/domestique» devient une sorte de clef de lecture de l'Outremer.
} 
faudrait parler de cohabitation, chez un même auteur, de préjugé et d'expérience. 